201

\section{RESTORING AKT ACTIVITY IN LATE OUTGROWTH ENDOTHELIAL PROGENITOR CELLS FROM HUMANS AT HIGH CARDIOVASCULAR RISK RESCUES THEIR ANGIOGENIC CAPACITY}

B Mercer, N Yuldasheva, H Viswambharan, V Baliga, S Stephen, J Askham, P Sukumar, A Skromna, R Mughal, H Imrie, M Gage, M Rakobowchuk, J Li, K Porter, S Ponnambalam, S Wheatcroft, D Beech, M Kearney, R Cubbon

\section{doi:10.1136/heartjnl-2013-304019.201}

Introduction Circulating endothelial progenitor cells may represent a useful autologous cell-based cardiovascular repair therapy; however, progenitor dysfunction is often noted in populations at high cardiovascular risk. We characterised the function of late outgrowth endothelial progenitor cells (LEPC) from south Asian (SA) and white European (WE) men using in vitro and in vivo angiogenesis assays, then set out to correct LEPC dysfunction by normalisation of molecular abnormalities relevant to angiogenesis.

Method Blood was collected from 12 healthy (WE) and (SA) men. In vitro angiogenesis was assessed via tube formation in response to VEGF-A on Matrigel; Western blotting was used to the assess Akt/ eNOS signalling axis. CMDil tracked LEPC were transfused into immunodeficient mice following femoral artery ligation induced hind limb ischaemia. Limb perfusion was serially assessed with Laser Doppler prior to harvesting the gastrocnemius muscle for quantification of CMDiI+/CD31+ neovessels using confocal microscopy. Lentiviruses were generated to express a constitutively active Akt1 mutant (E17KAkt), or enhanced green fluorescent protein (EGFP) as a control, in the SA LEPC. In vitro and in vivo angiogenesis studies were repeated in E17KAkt expressing SA LEPC. Data is presented as mean (standard error of mean) and $p<0.05$ is denoted by*.
Results Volunteers were well matched for age and cardiovascular risk factors, but the SA group were relatively insulin resistant (HOMA-IR $1.2[0.2]$ vs. $\left.0.5[0.1] \mathrm{au}^{*}\right)$. SA LEPC displayed impaired in vitro angiogenesis (1.9 [0.6] vs. 3.8 [0.5] tubular structures/microscopic field*) in comparison with WE LEPC. WE LEPCs augmented hind limb perfusion recovery, whereas either SA LEPC or vehicle did not (WE: 66.4 [4.7], SA: 49.3 [5.6], vehicle: 50.6 [2.8] \% ischaemic/non-ischaemic limb flux [Laser Doppler]; WE vs. SA*). This dysfunction was associated with a reduced concentration of phospho- S473-Akt (0.14 [0.05] vs. 0.81 [0.2] $\left.\mathrm{au}^{*}\right)$ and S1177-eNOS (0.05 [0.02] vs. 0.15 [0.01] au*). E17KAkt expressing SA LEPC demonstrated enhanced in vitro angiogenesis in comparison with EGFP expressing LEPC (2.4 [0.3] vs. 1.5 [0.2] tubular structures/microscopic field*); this was completely abrogated by the NOS inhibitor L-NMMA. E17Akt expressing LEPC rescued tissue perfusion following hind limb ischaemia (E17K: 63.4 [2.4] vs. EGFP 48.0 [1.6] \% ischaemic/non-ischaemic limb flux; E17K vs. EGFP*; E17K vs. WE non-transduced cells $\mathrm{p}=0.9$ ). Furthermore, E17KAkt expressing SA LEPC engrafted more frequently into neovessels within the ischaemic gastrocnemius muscle, compared with EGFP control (E17K: 22.6 [3.1], EGFP 7.4 [0.8] cells/mm2*).

Conclusions Dysfunction of LEPC derived from populations at increased cardiovascular risk is likely to impede their translation as a therapeutic strategy. This study provides proof of principle for the correction of LEPC dysfunction and mechanistically implicates altered signalling of the Akt1-eNOS signalling axis. 